Mots. Les langages du politique

\title{
Le climatoscepticisme : une approche interdiscursive
}

Climate denialism: an interdiscursive approach

El climatoescépticismo: un enfoque interdiscursivo

\section{Renaud Hourcade et Albin Wagener}

\section{OpenEdition \\ Journals}

Édition électronique

URL : https://journals.openedition.org/mots/28715

DOI : $10.4000 /$ mots. 28715

ISSN : 1960-6001

Éditeur

ENS Éditions

Édition imprimée

Date de publication : 18 novembre 2021

Pagination : $9-22$

ISBN : 979-10-362-0483-8

ISSN : 0243-6450

\section{Référence électronique}

Renaud Hourcade et Albin Wagener, "Le climatoscepticisme : une approche interdiscursive », Mots. Les langages du politique [En ligne], 127 | 2021, mis en ligne le 01 novembre 2021, consulté le 10 novembre 2021. URL : http://journals.openedition.org/mots/28715 ; DOI : https://doi.org/10.4000/ mots. 28715 


\section{Le climatoscepticisme : une approche interdiscursive}

Conforté par les gouvernements de Donald Trump aux États-Unis ou de Jair Bolsonaro au Brésil, voire à certains égards par celui de Boris Johnson au Royaume-Uni, le climatoscepticisme a connu ces dernières années des heures propices. L'espace politique que lui ont ouvert ces gouvernements confirme ce qui était déjà connu - l'étroite association entre le déni du réchauffement climatique et les idéologies conservatrices (Pottier, 2013) -, tout en invitant à prolonger la réflexion sur les facteurs qui permettent à cette opinion, dans ses diverses déclinaisons, de prospérer en dépit de l'établissement depuis maintenant plusieurs décennies d'un consensus scientifique extrêmement large sur le constat et les causes du réchauffement planétaire. Ce numéro thématique entend contribuer à cette réflexion en s'intéressant aux aspects interdiscursifs du climatoscepticisme. II propose, dans cette intention, de décaler le regard par rapport aux études portant sur les procédés rhétoriques et argumentatifs des discours et contre-discours climatiques - études désormais nombreuses et riches (par exemple Chetouani, 2007; Kurz et al., 2010 ; Washington, Cook, 2011 ; Elgesem et al., 2015 ; Fløttum, 2019; Fløttum et al., 2019). Les auteurs et autrices des quatre articles rassemblés dans ce numéro ont pris le parti d'explorer la manière dont les idées climatosceptiques sont représentées, entrecroisent d'autres thématiques, subissent des inflexions ou produisent des articulations rhétoriques dans un contexte d'interactions interdiscursive et intertextuelle avec d'autres types de discours publics, comme ceux de la presse écrite généraliste (Elise Schürgers), de la promotion de technologies liées au changement climatique (Johanna Gouzouazi), de la campagne politique britannique en faveur du Brexit (Alma-Pierre Bonnet) ou encore les débats qui se nouent sur le web à propos du véganisme (Albin Wagener).

Il nous semble qu'adopter ce regard éclaire certains ressorts sociolinguistiques du climatoscepticisme, en dessinant les contours d'un espace dialogique élargi de ce phénomène. Ce prisme fait ressortir un point saillant : le solide ancrage et les multiples résonances des contre-discours climatiques

Univ Rennes, CNRS, ARENES renaud.hourcade@cnrs.fr Université Rennes 2, INALCO albin.wagener@gmail.com 
au sein d'un univers discursif davantage articulé par une idéologie politique et un système de valeurs que par le souci de produire une critique scientifique raisonnée des connaissances climatiques. Selon les contextes nationaux, ces idéologies matricielles peuvent connaître des nuances et des degrés différents de polarisation. Elles varient aussi selon les groupes et les individus (Godard, 2012). Mais ces nuances n'empêchent pas de leur reconnaître un mécanisme commun : la perception des politiques climatiques et du consensus international qui les sous-tend comme une menace majeure, pesant sur des valeurs ressenties comme essentielles. Mesurer les résonances interdiscursives du climatoscepticisme permet de comprendre en quoi cette menace s'articule à d'autres sources d'inquiétude, comme le véganisme pour les consommateurs et producteurs de viande ou l'Union européenne pour les Brexiters, et de mesurer l'effet de cette articulation sur la circulation des discours. Une telle démarche, espérons-nous, pourra contribuer à expliquer le succès social d'une thèse pourtant battue en brèche dans le champ scientifique et par les sections dominantes du monde médiatique et politique - ce qui est précisément l'un des terreaux de son succès.

\section{Production et circulation des discours climatosceptiques}

Les liens étroits qui associent le déni du réchauffement climatique au mouvement conservateur ont été mis en évidence par les travaux en science politique, qui ont particulièrement mis l'accent surle cas des États-Unis (McCright, Dunlap, 2000 ; Dunlap, McCright, 2011; Oreskes, Conway, 2015). Il a été observé que la naissance et la promotion d'une «contre-science» du climat dans ce pays, au cours des années 1980, étaient le produit de la mobilisation de think tanks associés au parti républicain, ainsi que du lobbying de grandes entreprises, en particulier celles du secteur de la production pétrolière. Ce mouvement structuré, doté de ressources financières importantes, a répliqué avec succès les méthodes éprouvées dans la défense du tabagisme (Proctor, Schiebinger, 2008; Michaels, 2008) consistant à contester dans l'espace public et sur le terrain scientifique les résultats de travaux de recherche défavorables à leurs objectifs. Le but était - et demeure - de légitimer l'idée d'une incertitude sur l'évolution du climat et donc de retarder ou limiter l'action politique. Pendant plusieurs décennies, ces mouvements ont pris pour cible le travail du Groupe d'experts intergouvernemental sur l'évolution du climat (GIEC) mis sur pied par le Programme des Nations unies pour l'environnement (PNUE) et l'organisation météorologique mondiale en 1988, et plus globalement toute proposition internationale ou nationale visant à accroître la régulation des émissions de gaz à effet de serre liées à l'activité industrielle et économique. 
Même si cela peut paraître paradoxal à première vue, appréhender le discours climatosceptique comme un discours critique sur le réchauffement climatique n'est pas la meilleure manière de rendre compte de sa dynamique et de son succès. Certes, la négation de la réalité scientifique du phénomène de réchauffement planétaire a constitué le premier motif argumentatif des opposants aux travaux du GIEC, et promouvoir une science dite «alternative » reste un pilier de l'action des groupes d'intérêt (Cann, Raymond, 2018). Mais, depuis une vingtaine d'années, d'autres types d'arguments ont été mis en circulation : la contestation, non pas de la réalité, mais des causes du changement climatique, la remise en cause de l'évaluation modélisée de ses conséquences (Guillemot, 2014), ou encore la contestation de la nécessité d'agir et des modes d'action employés. Ces nuances rendent le climatoscepticisme éminemment multiforme du point de vue discursif. Le scepticisme sur les causes défend l'idée d'un réchauffement climatique non anthropique - selon ses tenants, il pourrait être dû aux grands cycles d'évolution du climat terrestre, à l'activité solaire ou à d'autres causes naturelles. Le scepticisme sur les conséquences estime que les modèles d'impact du réchauffement climatique sont erronés et souffrent d'une tendance à l'exagération'1. Il y aurait par ailleurs des aspects positifs au réchauffement non pris en compte par la «doxa » climatique, tels que la possibilité de mettre en culture des terres auparavant impropres à l'agriculture, ou encore des effets favorables sur la biodiversité. Un autre glissement argumentatif a consisté à ne plus contester les problèmes causés par le réchauffement climatique, mais à les relativiser en mettant en avant la capacité adaptative des sociétés humaines, leur génie technologique, ou le coût politico-économique jugé disproportionné des régulations adoptées, eu égard à d'autres priorités comme la lutte contre la pauvreté ou la promotion du libre-échange.

Si, à partir du milieu des années 1990, ces trois axes structurants du climatoscepticisme ont cohabité dans la sphère militante et lobbyiste (McCright, Dunlap, 2000), certains observateurs ont cru déceler un recul de la contestation de la science du climat à partir des années 2010, sous l'effet d'un consensus devenu difficile à déstabiliser (Boussalis, Coan, 2016, p. 96). Cependant, un examen approfondi montre que la contestation de la science, et en particulier des travaux du GIEC, tient toujours une place centrale dans la controverse. En 2010, l'institution onusienne a traversé une phase de turbulence à la suite de la révélation publique d'échanges d'emails internes et de l'identification d'une erreur (sur la perspective d'une fonte complète des glaciers himalayens à l’horizon 2035) dans le rapport de 2007, révélation que les cercles climatosceptiques ont tenté d'exploiter en imposant l'idée d'un «climategate». Plus largement, les études sociologiques sur le lobbying des think tanks américains

1. Voir par exemple Sylvie Brunel et Jean-Robert Pitte éd., Le ciel ne va pas nous tomber sur la tête: 15 grands scientifiques géographes nous rassurent sur notre avenir, Paris, JC Lattès, 2010. 
aboutissent également à la conclusion qu'il est trop tôt pour prononcer la fin du déni de la science, dans la mesure où ces derniers restent engagés dans un inlassable travail de sape sur ce plan (Boussalis, Coan, 2016; Cann, Raymond, 2018). Le débat public reste animé par des pseudo-arguments scientifiques dont la capacité de résistance à la réfutation étonne².

L'inlassable retour de cette controverse s'est traduit par une appropriation et une circulation du vocable climatosceptique comme mode de désignation signifiante de ces contestataires. L'article d'E. Schürgers, qui étudie la présence de ce lexème dans la presse francophone française et belge, offre un point de vue précisément documenté sur sa diffusion. L'autrice constate que la montée de son usage a suivi de près non pas la progression des connaissances sur le climat, mais l'émergence et le renforcement d'une action publique internationale. L'expression se répand en effet à partir de 2010, bien après la première phase de débat scientifique sur le réchauffement climatique, dans les années 1980 (Aykut et al., 2012; Guillemot, 2014). En d'autres termes, il n'y aurait vraisemblablement pas de climatoscepticisme en tant que formule (Krieg-Planque, 2009) s'il n'était question que de science du climat : c'est sa construction comme problème médiatique, à partir de 2002, et sa mise en politique de plus en plus consensuelle par la suite, qui ont réellement identifié comme telles les rhétoriques du déni et imposé leur étiquetage sous le vocable de climatoscepticisme. La formule pose un cadre pour le débat public et stabilise la charge polémique de l'objet climat. Au-delà de la trajectoire discursive de cette désignation, un angle de vue mériterait d'être évoqué : celui des bénéfices stratégiques qui lui sont associés. On peut voir en effet dans la diffusion du terme climatosceptique un avantage pour les opposants à la thèse d'un réchauffement anthropique, dans la mesure où le scepticisme renvoie positivement à la vertu heuristique du doute et de la controverse scientifique. La formule assoit donc une certaine respectabilité tout en écartant des étiquetages alternatifs moins favorablement connotés, tels que ceux associés à la notion de déni (plus fréquents en anglais sous la forme climate deniers) ou l'emploi de la formule plus dégradante encore de négationnistes. Ce déploiement sémantique n'est donc pas une petite victoire pour les climatosceptiques. Le prolongement de cette guerre de qualification pousse certains

2. Haydn Washington et John Cook les ont regroupés en cinq catégories : les théories du complot, le recours à de faux experts, le raisonnement fondé sur des attentes impossibles, la représentation faussée des connaissances, l'information sélective. Nombre d'arguments de cette controverse sont rebattus depuis une trentaine d'années, ce qui pousse les auteurs précités à les qualifier de «zombie arguments», car ils se relèvent sans cesse de leur tombe (Washington, Cook, 2011). L'argument d'un Groenland chaud et vert au Moyen Âge ou d'une climatologie qui annonçait dans les années 1970 une nouvelle ère glaciaire en sont des exemples. Comme l'observe Olivier Godard, le fait que, même battus en brèche sur le plan scientifique, ces arguments reviennent inlassablement dans les publications climatosceptiques témoigne du fait que leurs auteurs ne cherchent pas à participer au débat scientifique, mais à conquérir l'opinion publique (Godard, 2012). 
climatosceptiques à se présenter aujourd'hui comme des climatoréalistes, une expression connotée à la fois par les vertus du bon sens et par un certain pragmatisme politique. À l'inverse, les cercles climatosceptiques ont multiplié les formules pour stigmatiser et affaiblir le consensus sur le climat, en évoquant régulièrement une idéologie réchauffiste ou des thèses climato-alarmistes.

C'est aussi pour mesurer les évolutions de l'environnement médiatique dans lequel les climatosceptiques promeuvent leurs arguments que l'article d’E. Schürgers s'avère précieux. Dans les années 1990, les médias généralistes ont offert aux climatosceptiques une caisse de résonance sans commune mesure avec leur représentativité scientifique. Les travaux qui se sont intéressés à ce phénomène y ont vu un effet de l'éthique professionnelle des journalistes, qui les aurait poussés à mettre en valeur la pluralité des points de vue sur ce sujet comme surn'importe quel autre (Boykoff, Boykoff, 2004). En France, dans les grands médias, la décennie 2000, qui est celle de l'institutionnalisation de la lutte contre le changement climatique, n’a pas été favorable à la circulation des discours climatosceptiques, comme en témoigne, par exemple, leur absence quasi totale des journaux télévisés et des grands titres de presse (Aykut et al., 2012; Comby, 2012). Les années 2010 leur sont à peine plus propices et si, selon E. Schürgers, l'expression de climatosceptique achève de se banaliser à cette époque, elle accompagne avant tout un traitement critique, voire une forme de lassitude, dans la couverture de ce thème par les grands médias. Mais paradoxalement, ce large discrédit n'empêche pas que s'impose en bruit de fond l'idée qu'il existe une controverse sur les sciences du climat, bruit de fond dont la banalisation du lexème dans la «grande presse» est un bon indicateur. Les contributions du présent numéro le démontrent également. En France, la circulation des thèses climatosceptiques est entretenue pour l'essentiel en dehors des grands médias traditionnels, au travers de livres accrocheurs et de la couverture bienveillante qui leur est offerte par certains titres de presse tentés de se détacher du consensus «dogmatique» sur ce sujet3. Le résultat est qu'en dépit de l'accumulation d'arguments en faveur de la thèse d'un réchauffement climatique anthropique préoccupant, les thèses

3. 2009-2010 est une période charnière pour la circulation et le renforcement des postures climatosceptiques. D’une part le climategate donne quelques munitions médiatiques aux dénonciateurs de la science du climat internationale incarnée par le GIEC. D'autre part les livres de dénonciation du « dogme réchauffiste » se sont multipliés ces années-là dans la sphère francophone. On peut citer par exemple Vincent Courtillot, Nouveau voyage au centre de la Terre (2009), Claude Allègre, L'imposture climatique ou La fausse écologie (2010), Benoît Rittaud, Le mythe climatique (2010). Claude Allègre tenait cependant des chroniques climatosceptiques dans L'Express dès 2006. Selon Jean-Baptiste Comby, cette présence accrue - mais qui reste marginale - des climatosceptiques dans les médias au début des années 2010 est aussi la conséquence d'une transformation de l'approche journalistique du sujet, désormais devenue transversale dans les rédactions et qui n'est donc plus seulement l'apanage de spécialistes des rubriques sciences ou environnement, dont la couverture du sujet était plus en phase avec le discours scientifique dominant (Comby, 2012). 
sceptiques n'ont finalement jamais cessé d'être représentées et véhiculées dans le champ médiatique, a minima grâce aux formidables caisses de résonance offertes par les réseaux sociaux. Certes, des travaux récents suggèrent que le souci de l'équilibre qui guidait le traitement médiatique jusqu'aux années 2000 a nettement reflué à mesure que s'affermissait la perception d'un consensus politique (Schmid-Petri et al., 2015). Mais ce qui ressort de l'étude des médias généralistes mériterait d'être contrebalancé par une étude plus attentive et systématique de médias tels que, en France, CNews ou Sud Radio, ou en presse écrite Le Point ou Valeurs actuelles, pour lesquels les sujets environnementaux peuvent servir de repère idéologique conservateur et alimenter une ligne éditoriale en quête de clivages sociaux et politiques. Loin de la lassitude des «grands» médias, ces titres et stations offrent depuis vingt ans de multiples tribunes aux tenants du « réalisme » climatique. Il serait tout aussi intéressant de mesurer le contraste entre la progressive mise à l'écart des discours sceptiques dans les médias légitimes et leurvigueur maintenue (voire croissante) dans certains espaces du web et des réseaux sociaux. C'est en effet sur ces réseaux que les communautés conservatrices se structurent et s'engagent autour des discours climatosceptiques (Jylhä et al., 2016; Bloomfield, Tillery, 2019) - des discours qui peuvent également toucher les individus les plus libéraux (Washburn, Skitka, 2017).

\section{Les cadres moraux du déni}

Antonin Pottier avait identifié il y a quelques années plusieurs caractéristiques des discours climatosceptiques, qui invitaient à les rapprocher des discours réactionnaires, au sens d'Albert O. Hirschman (Pottier, 2013; Hirschman, 1991). Les articles rassemblés dans ce numéro confortent cette observation selon un autre point de vue, celui des interrelations entre discours sceptiques et autres types de discours politiques marqués par des idéologies conservatrices. Traquer l'affleurement de rhétoriques climatosceptiques au sein des débats sur le Brexit (A.-P. Bonnet) ou sur le véganisme (A. Wagener) se révèle très éclairant de ce point de vue. Le souverainisme britannique exacerbé au moment de la campagne sur le référendum sur la sortie de l'Union européenne ou la réaffirmation de la pensée spéciste comme socle culturel des sociétés occidentales ne touchent pas à la physique de l'atmosphère, mais relèvent de systèmes de valeurs profondément enracinés. Ces systèmes de valeurs sont au fondement des sociétés occidentales. La révolution industrielle, en particulier à partir de l'exploitation pétrolière à grande échelle, a permis le développement d'un mode de domination viriliste de la nature (Dagget, 2019), associé à un ethnocentrisme nationaliste et à une confiance absolue dans le progrès des sciences et des techniques. Ces différents éléments composent un système 
indissociablement économique, moral et culturel qui a structuré la prospérité de l'Occident et l'expansion de sa puissance, au grand détriment d'autres sociétés. Or, de même que des revendications sociales comme celles du féminisme, du postcolonialisme, des minorités racisées ou sexuelles peuvent être combattues par les mouvements conservateurs parce qu'elles menacent les fondements idéologiques de cette domination, le constat de plus en plus consensuel d'un réchauffement climatique causé par l'activité (économique) anthropique est porteur d'une remise en cause brutale de ce modèle de société (Bulkeley, 2001; Guillemot, 2014).

Dès lors, à la faveur d'une polarisation politique de plus en plus prégnante dans de nombreux pays, la question d'accepter ou non la "thèse» du réchauffement climatique est devenue, comme de nombreux autres enjeux environnementaux, un objet de positionnement identitaire et moral extrêmement structurant (Kurz et al., 2010; McCright, Dunlap, 2011; Chinn et al., 2020). Le «monde à défendre» face à cette remise en question 4 peut prendre des formes variables, ce qui donne aux climatosceptiques un caractère relativement composite, particulièrement en France où ils apparaissent faiblement organisés (Guillemot, 2014). Néanmoins, ils convergent dans le fait de se déterminer sur la base de récits interprétatifs fondés avant tout sur des valeurs, récits qui leur permettent d'appréhender aisément un sujet complexe : soit la lutte contre le changement climatique est compatible avec le projet politique à leurs yeux prioritaire, soit elle le contrarie. Il en résulte un cercle de confirmation/ disqualification mécanique, reposant largement sur les affects et imaginaires identitaires, qui consiste à percevoir dans la lutte contre le réchauffement climatique, et plus globalement dans toute proposition de régulation environnementale, une menace existentielle5. Par suite, la lutte contre le réchauffement climatique, a fortiori si elle est défendue dans des instances internationales jugées illégitimes ou à la solde de puissances rivales, s'en trouve conçue comme une agression, selon les cas et les nuances, contre la liberté, le capitalisme, la démocratie ou le progrès - ou contre tout cela à la fois.

4. Riley E. Dunlap et Aaron M. McCright rassemblent les différentes nuances de cette posture sous le vocable d'anti-réflexivité, expression par laquelle ils désignent « une opposition véhémente aux forces de la modernisation réflexive (par exemple la science de l'environnement et le mouvement écologiste) visant à défendre le système capitaliste industriel moderne de toute remise en cause» (McCright, Dunlap, 2012).

5. Ce positionnement pousse certains climatosceptiques à tenter d'entretenir des controverses sur des aspects encore moins sujets à débats que le réchauffement climatique, comme l'impact sanitaire de la qualité de l'air. Voir à ce sujet Christian Gérondeau, L'air est pur à Paris mais personne ne le sait, L'Artilleur, 2018, dans lequel l'auteur, un polytechnicien, ancien ingénieur né en 1938 et auteur de plusieurs ouvrages climatosceptiques, «dénonce les stratagèmes, mensonges et forfaitures que mettent en œuvre les services officiels de l'environnement non seulement pour cacher aux Français que l'air des principales grandes villes de France est désormais pur mais surtout pour leur faire croire que la pollution s'accroît». 
Ainsi, dans l'esprit de lobbyistes conservateurs américains, à l'image d'un Fred Singer actif depuis l'époque de la guerre froide (Oreskes, Conway, 2015), l'écologie n'est rien d'autre que l'habit neuf du communisme, le contre-modèle par excellence. Cette politisation agonistique permet aux cercles conservateurs d'identifier clairement leur camp - celui du small state et de la libre-entreprise, du pragmatisme et de la confiance dans les technologies. Dans le cas français, comme le relève Hélène Guillemot (2014), le spectre communiste est sans doute moins prégnant. Le combat contre l'écologie recoupe en revanche souvent une «défense des valeurs scientistes et productivistes» (Guillemot, 2014, p. 347), qui place dans les solutions technoscientifiques des promesses d'émancipation et de progrès collectifs. À l'image de Claude Allègre, un certain nombre d'intellectuels et de scientifiques sont attachés à cette croyance, comme à sa traduction politique dans une forme de jacobinisme techno-industriel, qu'ils associent volontiers à l'identité et au rayonnement de la France. L'un des effets les plus importants de cette polarisation politique est sans doute d'avoir favorisé la circulation et la reprise des thèses climatosceptiques ainsi que leur promotion bien au-delà des think tanks d'économistes libéraux et de grandes entreprises qui, aux États-Unis particulièrement, en ont fait initialement la promotion. Plus encore, alors que ces derniers ont souvent pris le virage d'une reconnaissance du réchauffement climatique global et promeuvent en conséquence des stratégies d'entreprise "verdies» (à l'image des objectifs de décarbonation de l'activité de groupes comme Total ou Engie), la combativité climatosceptique semble se manifester désormais sous la forme d'une guerre de tranchées culturelle, livrée par l'intermédiaire de supports médiatiques de plus en plus balkanisés selon leur tendance politique. R. E. Dunlap et Peter J. Jacques l'observent également dans une enquête sur la production de livres climatosceptiques aux États-Unis : les think tanks conservateurs en produisent beaucoup moins, tandis qu'un grand nombre de ces ouvrages sont désormais écrits par des profanes, sans compétence technique particulière sur le climat, mais qui se proclament conservateurs et «dont on peut supposer qu'ils ont adopté une position de déni du changement climatique parce que cette position est devenue un principe central du conservatisme» (Dunlap, Jacques, 2013).

Pour résumer, de pragmatique et stratégique à l'origine, le climatoscepticisme contemporain est devenu intensément idéologique : il n'est plus l'affaire des grandes majors pétrolières, mais celle de conservateurs chauffés à blanc par le sentiment d'une menace existentielle et emportés par ce que l'on appelle des «paniques morales» (Cohen, 1972). Notons tout de même que cette base climatosceptique demeure alimentée, aux États-Unis et dans quelques pays, par des institutions puissantes comme des grands think tanks conservateurs, des cabinets de relations publiques ou des leaders politiques, tel Donald Trump. En Europe et en France, elle est davantage stimulée par des francs-tireurs, situés pour la plupart très en marge du système médiatique et 
politique, qui s'expriment pour l'essentiel dans des médias « de niche», mais dont l'audience, l'intérêt et la capacité à entretenir l'idée d'une polémique ne sont pas négligeables. Ils y œuvrent notamment à travers le recours à une rhétorique tout à la fois victimaire et héroïque, en se présentant comme les victimes courageuses d'une idéologie hégémonique et intolérante à la critique. Une doxa incarnée par exemple à leurs yeux par l'activiste Greta Thunberg, qui fut violemment prise pour cible en 2019, lors de sa venue à l'Assemblée nationale, notamment de la part de personnages comme le philosophe Michel Onfray ou l'éditorialiste Laurent Alexandre (Wagener, 2020).

\section{Le climatoscepticisme dans l'espace des discours conservateurs}

Comme nous l'avons déjà précisé, le climatoscepticisme constitue un phénomène résolument interdiscursif, au sens où il peut être réellement embrassé à partir du moment où ses incarnations discursives sont étudiées dans toutes leurs diversités. C'est grâce à ce type d'étude polydimensionnelle que l'interdiscours peut alors être cerné et envisagé en tant que processus complexe (Garric, Longhi, 2013) et relié aux études systémiques6 ${ }^{\text {(Wagener, 2019). }}$ Comme d'autres phénomènes discursifs issus des univers conservateurs ou réactionnaires, le climatoscepticisme joue sur des contraintes linguistiques et cognitives de sélection contextuelle (Maillat, 2013) pour ne conserver que les arguments ou les faits qui servent le discours. Ce faisant, il participe à la reconstruction d'une réalité basée sur une vision idéologique des faits (Kopytowska, 2015) qui vise à contourner la prise en considération des études et des réalités concernant le changement climatique, pour les croiser avec d'autres registres discursifs qui ne partagent pas la scientificité des études du GIEC.

Sur le terrain de l'ingénierie climatique, par exemple, J. Gouzouazi montre comment certains think tanks font subtilement évoluer leur positionnement sur le terrain de la science climatique pour orienter la prise de décision dans un sens qui leur soit favorable. En opérant une confusion volontaire entre processus de recherche scientifique et technicité de l'ingénierie, ceux-ci promeuvent en fait une dépolitisation des enjeux climatiques pour les remettre sous la coupe des solutions techniques. Cependant, cette dépolitisation est en fait une repolitisation visant à préserver les intérêts économiques et financiers associés au mode traditionnel d'exploitation de la nature. Cette stratégie discursive amène les auteurs de ces think tanks à revêtir les habits de ceux qui agissent pour contrer le réchauffement climatique, tout en souhaitant prioritairement maintenir leur domination sociale et économique dans un monde qui ne doit

6. Ici, les études systémiques sont envisagées au sens où elles articulent théorie de la complexité et méthodologie d'analyse du discours, dans une dimension à la fois linguistique, sémantique, sociale, interactionnelle et multimodale. 
pas changer - une attitude commune à de nombreux think tanks néolibéraux (Neubauer, 2011). Dans un autre contexte, A.-P. Bonnet montre à quel point les discours climatosceptiques ont réussi à s'inscrire dans le moment politique du Brexit, qui constitue en soi un véritable moment discursif (Moirand, 2007) - soit un moment temporel de polarisation topique et argumentative spécifique, délimité par des événements particuliers. De fait, la manière dont les discours climatosceptiques se sont invités dans la campagne politique qui préparait le Brexit au Royaume-Uni rappelle à quel point la dimension argumentative du discours est centrale pour comprendre dans quelle mesure la langue impacte la circulation des idéologies, les prises de position politique et, in fine, toutes les autres thématiques sociales (Amossy, 2011). Une nouvelle fois, au Royaume-Uni, l'entrée par l'interdiscours montre à quel point le climatoscepticisme est irrigué par d'autres prises de position politiques qui n'ont pas nécessairement grand-chose à voir avec les thématiques climatiques. Ici, A.-P. Bonnet évoque notamment le glissement sémantique et lexical conduisant du Brexit au Clexit, autre mot-valise qui dépeint l'urgence climatique comme un dogme religieux imposé à toutes et à tous au détriment de la souveraineté et de l'économie britanniques, et dont il serait urgent de sortir - l'analogie avec l'Union européenne n'a, ici, bien entendu, rien de fortuit. Enfin, dans le cas de la circulation des discours en ligne, $A$. Wagener s'intéresse à la manière dont les discours anti-véganes peuvent, en partie, renfermer des éléments climatosceptiques; ceci est notamment justifié par le fait que la réduction de la consommation de produits carnés est régulièrement citée par les spécialistes du climat, tant ceux-ci pèsent sur les émissions de gaz à effet de serre dans le monde. II remarque que les discours anti-véganes consacrent beaucoup d'efforts à justifier l'acte de consommation de viande, en le situant principalement sur le plan anthropologique, culturel, affectif, mais parfois également, en référence au débat sur le réchauffement climatique, sur le terrain scientifique. Dans cette optique, le climatoscepticisme n'intervient pas comme un courant de pensée qui motiverait des comportements, mais se manifeste comme une production argumentative contextualisée, destinée à justifier un comportement en venant en renfort d'autres registres.

Ainsi donc, les contributions du présent numéro de la revue Mots. Les langages du politique permettent de mettre en lumière plusieurs caractéristiques du climatoscepticisme. Tout d'abord, il peut apparaître dans bien des discours sans y occuper une place centrale. Il y joue un rôle d'argumentaire complémentaire ou connexe, qui entre en résonance avec l'idéologie qui irrigue les énoncés des locutrices et des locuteurs. Mis en scène comme un ajout argumentatif, le climatoscepticisme semble voué à ce rôle secondaire, peutêtre en raison du fait que le consensus concernant le climat semble ne plus laisser de réelle place à l'expression du doute, en dehors des rares groupes qui s'en sont fait une spécialité militante. Le second enseignement, et non des 
moindres, indique que le climatoscepticisme est susceptible de s'inviter dans un nombre apparemment assez varié de thématiques. Mobilisé sous la forme de fragments discursifs climatosceptiques, il peut être associé à une grande diversité de sujets de société - qu'il s'agisse de nos assiettes, de débats politiques nationaux, d'innovation technologique ou de productions de presse ou de réseaux sociaux. En d'autres termes, peu importent les dispositifs et les sujets : puisque le changement climatique est précisément un phénomène systémique qui questionne l'ensemble de nos modes de vie et choix de société, le climatoscepticisme se retrouve, en retour, mobilisé pour accompagner une pluralité de prises de position. D'une certaine façon, les fragments de discours climatosceptiques peuvent être analysés comme autant de poches de résistance anthropologique, culturelle et sociale, face à un phénomène qui interroge l'intégralité de notre rapport au monde et aux autres. Dans une certaine mesure, ces fragments discursifs climatosceptiques sont autant d'indices que l'urgence climatique est bel et bien perçue par toutes et tous, en lien avec un nombre indéterminable de sujets et de thématiques, et que cette prise en compte est ressentie comme menaçante par bon nombre de citoyennes et de citoyens. Elle est ressentie comme telle, car elle impose à toutes et à tous une prise de conscience rapide, des actions dans l'urgence, et surtout des renoncements considérables auxquels il peut être particulièrement difficile de s'affronter (Norgaard, 2011). Cela étant dit, les discours climatosceptiques ne doivent pas être uniquement considérés comme un phénomène anthropologique spontané d'autodéfense; comme nous l'avons souligné, ils ont été et demeurent largement nourris par des groupes d'intérêt économiques, politiques et financiers, qui ont pour objectif de préserver leur domination et qui entretiennent dans ce but un double mouvement de mésinformation et de politisation.

\section{Références}

Amossy Ruth, 2011, «Des sciences du langage aux sciences sociales : l'argumentation dans le discours ", A contrario, $\mathrm{n}^{0} 16, \mathrm{p} .10-25$.

Aykut Stefan, Сомвy Jean-Baptiste, GuILlemot Hélène, 2012, "Climate change controversies in French mass media 1990-2010 ", Journalism Studies, vol. XIII, n² 2, p. 157174 .

Bloomfield Emma Frances, Tillery Denise, 2019, "The circulation of climate change denial online: rhetorical and networking strategies on Facebook», Environmental Communication, vol. XIII, nº 1, p. 23-34.

Boussalis Constantine, CoAN Travis G., 2016, "Text-mining the signals of climate change doubt», Global Environmental Change, vol. XXXVI, p. 89-100.

BоyкоғF Maxwell T., Boykoff Jules M., 2004, «Balance as bias: global warming and the US prestige press », Global Environmental Change, vol. XIV, nº 2, p. 125-136. 
BULKELEY Harriet, 2001, « Governing climate change: the politics of risk society? », Transactions of the Institute of British Geographers, vol. XXVI, nº 4, p. 430-447.

CANN Heather W., RAYMond Leigh, 2018, «Does climate denialism still matter? The prevalence of alternative frames in opposition to climate policy », Environmental Politics, vol. XXVII, n³ 3, p. 433-454.

Coren Stanley, 1972, Folk Devils and Moral Panics: The Creation of the Mods and Rockers, Londres, MacGibbon and Kee.

ChetouAnI Lamria, 2007, «Les mots de la controverse sur le changement climatique», Le Télémaque, n³1, p. 81-104.

Chinn Sedona, Hart P. Sol, Soroka Stuart, 2020, "Politicization and polarization in climate change news content, 1985-2017», Science Communication, vol. XLII, nº 1 , p. 112-129.

CомBY Jean-Baptiste, 2012, «Les médias face aux controverses climatiques en Europe: un consensus fragilisé mais toujours structurant», dans E. Zaccaï, F. Gemenne, et J.-M. Decroly éd., Controverses climatiques, sciences et politique, Paris, Presses de Sciences Po, p. 157-171.

DagGet Cara New, 2019, The Birth of Energy: Fossil Fuels, Thermodynamics, and the Politics of Work, Durham, Duke University Press.

DUNLAP Riley E., JACQUES Peter J., 2013, «Climate change denial books and conservative think tanks: exploring the connection», American Behavioral Scientist, vol. LVII, no 6, p. 699-731.

DUnlAP Riley E., MCCRIGHT Aaron M., 2011, «Organized climate change denial», dans J.S. Dryzek, R. B. Norgaard et D. Schlosberg éd., The Oxford Handbook of Climate Change and Society, Oxford, Oxford University Press, p. 144-160.

Elgesem Dag, Steskal Lubos, Diakopoulos Nicholas, 2015, «Structure and content of the discourse on climate change in the blogosphere: the big picture », Environmental Communication, vol. IX, nº 2, p. 169-188.

FLØтtum Kjersti éd., 2019, The Role of Language in the Climate Change Debate, New York, Routledge.

FLøTTUM Kjersti, GJERSTAD Øyvind, Oloko Francis Badiang, 2019, « Les voix dans le discours climatique : essai d'une combinaison de la polyphonie avec l'analyse de contenu, l'analyse narrative et l'analyse rhétorique», Cahiers de praxématique, $n^{0} 73$, https://doi.org/10.4000/praxematique.5747 (consulté le 12 juillet 2021).

GARRIC Nathalie, LONGHI Julien, 2013, «Atteindre l'interdiscours par la circulation des discours et du sens», Langage et société, $\mathrm{n}^{0}{ }_{144}$, p. 65-83.

GodARD Olivier, 2012, "Les controverses climatiques en France», dans E. Zaccaï, F. Gemenne, et J.-M. Decroly éd., Controverses climatiques, sciences et politique, Paris, Presses de Sciences Po, p.117-140.

GuILLEMOT Hélène, 2014, "Les désaccords sur le changement climatique en France : audelà d'un climat bipolaire », Natures Sciences Sociétés, vol. XXII, nº 4, p. 340-350.

HIRSCHMAN Albert O., 1991, Deux siècles de rhétorique réactionnaire, P. Andler trad., Paris, Fayard. 
Jylhä Kirsti, Cantal Clara, Akrami Nazar, Milfont Taciano, 2016, «Denial of anthropogenic climate chance: social dominance orientation helps explain the conservative male effect in Brazil and Sweden », Personality and Individual Differences, vol. XCVIII, p.184-187.

KoprtowsKa Monika, 2015, "Mediating identity, ideology and values in the public sphere: towards a new model of (constructed) social reality », Lodz Papers in Pragmatics, vol. XI, n² 2, p. 133-156.

KRIEg-PlAnque Alice, 2009, La notion de "formule» en analyse du discours : cadre théorique et méthodologique, Besançon, Presses universitaires de Franche-Comté.

Kurz Tim, Augoustinos Martha, СRABв Shona, 2010, "Contesting the "national interest" and maintaining "our lifestyle": a discursive analysis of political rhetoric around climate change », British Journal of Social Psychology, vol. XLIX, nº 3, p.601625.

MaILlat Didier, 2013, "Constraining context selection: on the pragmatic inevitability of manipulation », Journal of Pragmatics, vol. LIX, Part B, p. 190-199.

MCCRIGHT Aaron M., DunlaP Riley E., 2012, «Déni organisé et polarisation politique autour des changements climatiques aux États-Unis», V. Lacroix trad., dans E. Zaccaï, F. Gemenne, et J.-M. Decroly éd., Controverses climatiques, sciences et politique, Paris, Presses de Sciences Po, p. 173-194.

- 2011, "The politicization of climate change and polarization in the American public's views of global warming, 2001-2010 », The Sociological Quarterly, vol. LII, no 2, p. 155-194.

- 2000, «Challenging global warming as a social problem: an analysis of the conservative movement's counter-claims ", Social Problems, vol. XLVII, nº 4, p. 499-522.

Michaels David, 2008, Doubt is Their Product: How Industry's Assault on Science Threatens your Health, Oxford, New York, Oxford University Press.

MoIRAND Sophie, 2007, Les discours de la presse quotidienne : observer, analyser, comprendre, Paris, PUF.

Neubauer Robert, 2011, «Manufacturing junk: think tanks, climate denial, and neoliberal hegemony ", Australian Journal of Communication, vol. XXXVIII, nº 3, p. 65-88.

NorgAARD Kari Marie, 2011, Living in Denial: Climate Change, Emotions, and Everyday Life, Cambridge, MIT Press.

ORESKes Naomi, ConwaY Erik M., 2015, Merchants of Doubt: How a Handful of Scientists Obscured the Truth on Issues from Tobacco Smoke to Global Warming, New York, Bloomsbury Press.

PotTIER Antonin, 2013, "Le discours climato-sceptique : une rhétorique réactionnaire », Natures Sciences Sociétés, vol. XXI, nº 1, p. 105-108.

Proctor Robert N., SChiebinger Londa éd., 2008, Agnotology: The Making and Unmaking of Ignorance, Stanford, Stanford University Press.

Schmid-Petri Hannah, Adam Silke, Schmuckı Ivo, HäUSSLER Thomas, 2015, «A changing climate of skepticism: the factors shaping climate change coverage in the US press », Public Understanding of Science, vol. XXVI, nº 4, p. 498-513. 
WAGENER Albin, 2020, "Internet contre Greta Thunberg : une étude discursive et argumentative», Argumentation et analyse du discours, no25, https://doi. org/10.4000/aad.4747 (consulté le 12 juillet 2021).

- Discours et système : théorie systémique du discours et analyse des représentations, Bruxelles, Peter Lang.

WASHBURN Anthony, SKITKA Linda, 2017, «Science denial across the political divide: liberals and conservatives are similarly motivated to deny attitude-inconsistent science ", Social Psychological and Personality Science, vol. IX, nº 8, p. 972-980.

Washington Haydn, Соok John, 2011, Climate Change Denial: Heads in the Sand, Londres, Earthscan. 